\title{
Cost Effective Approach for Object Sorting
}

\author{
J. D. Gavade \\ Dept.of Electronics \\ Textile \& Engg Institute, \\ Ichalkaranji,India
}

\author{
P. K. Kharat \\ Dept. of I.T. \\ Walchand College of \\ Engg,Sangli,India
}

\author{
S. K. Laga \\ Dept.of Electronics \\ Textile \& Engg Institute, \\ Ichalkaranji,India
}

\begin{abstract}
Modernization, although, has led into the development in terms of sophistication of human efforts in bringing out massproduction of more precisely manufactured goods; yet it in some ways it needs to be modified to be a smarter intelligent system. Intelligence in terms of decision making ability of machines, more specifically, robots. Modern day needs have broken the traditional belief of human interference just as an instructor to a machine or robot, and it is leaping towards an era demanding self motivated and corrective decision-maker automations. In many situations, autonomous systems do provide effective solutions to menial or dangerous tasks. In many cases, it is desirable to design an automated system that can identify objects and relocate them if the object meets certain prescribed criterion. This paper presents a similar but simplified system which will sort the objects according to different parameters such as color and size using simple image processing technique. The system is trained initially with a set of images defined as the reference images. Then while execution, real time images are compared with the reference image parameters and respective output is then fed to the placing system through parallel port of the computer.
\end{abstract}

\section{Keywords}

Autonomous systems; decision-maker; gigantic tasks; massproduction; precisely manufactured; repetitive; self motivated; sophistication

\section{INTRODUCTION}

Today we are moving towards a world of automation and intelligent systems. The applications of electronics are not only limited to computers and communications, they are also influencing every field. In this day and age of computers, automation is becoming sensing, monitoring and storing the changes per millisecond involved in experiment with accuracy. Moreover, the repetitive tasks with same accuracy and sensitivity can be completed using automated instruments.

Normally, sorting of the objects is done by manually. It consists of 4 integrated stations called distribution, testing, processing and handling. Old sorting method uses a set of inductive, capacitive and optical sensors do differentiate object color in the testing station. Handling is done by using a programmed manipulator. No vision capability exists in the system to improve its performance and flexibility. In this case, there is a possibility of minor error which will affect the accuracy in sorting. Also for huge systems, time and manpower required will e very high. Automated systems can be used to remove such human errors and also it saves time and money. [1][8]

Presently there is a system which can sort the Ball-Bearings of Different sizes which is done by the Texas Instrument. In that particular system the Ball-Bearings of different sizes are sorted according to their program developed in LAB-VIEW ${ }^{\circledR}$. But this sorting systems tend to include the development of an electronic weight system which is not comfortable in every field so we are choose to implement vision based sorting which may measure size, with a friendly user interface that enables definition of classification parameters, reconfiguration of the outputs and maintenance of production statistics.[2]

One of the system which is used for sorting is based on weight comparison is currently used in RBI (Reserve Bank of India). This type of sorting is used for separation of coins. Some commercially available systems are approaching this objective, but this weighting method is not suitable everywhere in the industry.

Most of the systems we can find in the market are based on special architectures, for instance, DSP-based processors boards, hardware implementation of special purpose algorithm, VME architectures, etc. These DSP-processor based systems are too costly for small-scale industries. Hence the need for building a cost effective and user friendly system was felt.

The objective of this paper is to explain the schematic of the design, development and implementation of a robust and unique conveyor system employed in the developed machine vision system for sorting of the objects. [3].

The developed system mainly consists of three major parts, namely Conveyor system, imaging system and Computer system. The mechanical assembly of the system consists of mainly four sections: 1) Object feeding section, 2) Object Orienting section,

3) Object imaging section and 4) Object sorting section.

\section{SYSTEM DESIGN AND METHODOLOGY}

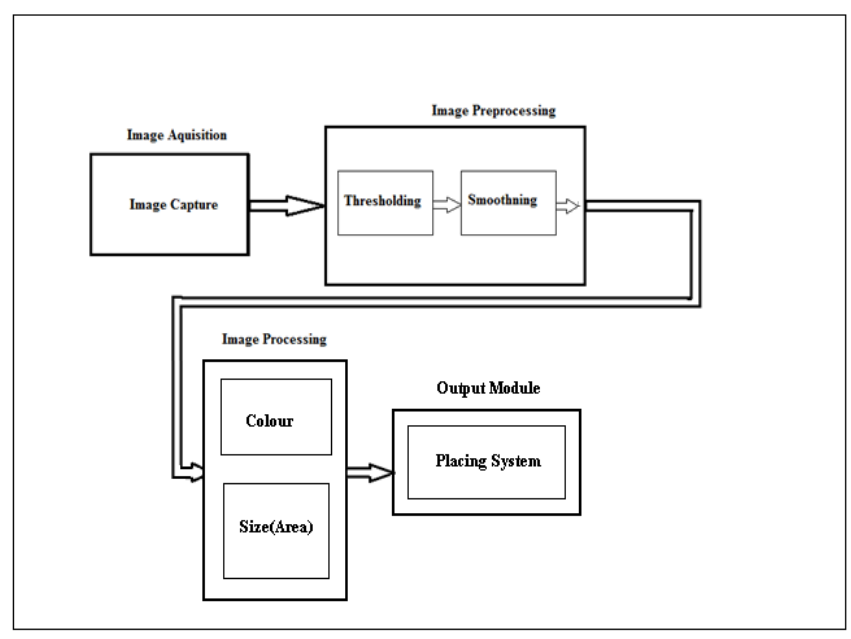

Fig 2.1: System Block Diagram

The main aim is to sort the objects on various parameters like color and size. So the proposed block diagram consists of following blocks-

A. Image Acquisition

B. Image Processing

C. Output Module 


\subsection{Image Acquisition}

To work with the image one acquires, one must bring it into the MATLAB $^{\circledR}$ workspace first. To bring the image into MATLAB ${ }^{\circledR}$ workspace image acquisition toolbox is used. The image of the object is captured using camera mounted on the computer. The input image from the camera cannot be given for processing directly. Pre-processing is done on the image such as thresholding. Then only object image I converted in binary format. Then this binary image has to be smoothened so that paper and salt noise can be removed. This final threshold image of object is now ready for processing. [4]

\subsection{Image Processing}

Here the objects are sorted with the help of two parameters color and size. There are two codes for calculating size and identifying color object. In calculating size the sum command is used to find out number of white pixels present in the frame. To identify the color, firstly the R, G, B contents are separated and by using same command the color is identified. [4]

\subsection{Output Module}

The output module consists of the conveyor assembly and stepper motor assembly. After calculating the size and identifying the color, command will be sent to direct the stepper motor through parallel port of the computer. Conveyor assembly is continuously in $\mathrm{ON}$ state. According to the size and color the stepper motor assembly is directed. [5]

\section{HARDWARE DESIGN TECHNIQUE}

In this section we focus on the hardware configuration of the system.
A. Interfacing of camera
B. Interfacing of placing system
C. Power supply design

\subsection{Interfacing of camera}

12 megapixel night-vision camera which is having USB interfacing facility is used to capture the image. The camera is directly interfaced to USB port. Interfacing of placing system

\subsection{Interfacing of Placing system}

There are two assembly of placing systems:

3.2.1. Conveyor assembly: It consists of two Bushing Bearings which are used to minimize the friction torque. The conveyor belt is made up of the black cloth (5218) to create the constant background for thresholding. Two DC Gear Motors are used to run the conveyor assembly. The DC Gear Motor has following features:

- $12 \mathrm{v}$ DC

- $\quad 750 \mathrm{~mA}$ max current

- 45 RPM

3.2.2. Stepper motor with driver circuit assembly: It consists of the stepper motor with its driver circuit. Following are the specifications of the stepper motor.

- $12 \mathrm{v} \mathrm{dc}$.

- $\quad 0.67 \mathrm{~A}$ per phase

- 7 kg-m Torque

- $\quad 1.8$ Degree per step

- 5-wire (two phase)

3.2.3 Driver circuit: The driver circuit is used to drive the stepper motor through parallel port of computer. The output of driver circuit is $12 \mathrm{v}$ de with output current 1.2A. The main aim of the driver circuit is to protect the stepper motor from peak current pulse (i.e. short circuit). [5]

\subsection{Power supply design}

Fot his system,two DC supplies are reuired.

- $\quad 12 \mathrm{v}$ with $750 \mathrm{~mA}$ current

- $12 \mathrm{v}$ with $1.2 \mathrm{~A}$ current

3.3.1 12v with 750mA current: In this supply we are used regulator IC LM7812 which has following specifications:

- Constant 12v DC

- $\quad 750 \mathrm{~mA}$ max current

- Output current in excess of $1 \mathrm{~A}$

- Internal short circuit current limit

- Internal thermal overload protection

The output of this power supply is $12 \mathrm{~V}$ with $750 \mathrm{~mA}$ current. This is used to drive the Gear motors which are in parallel. [5]

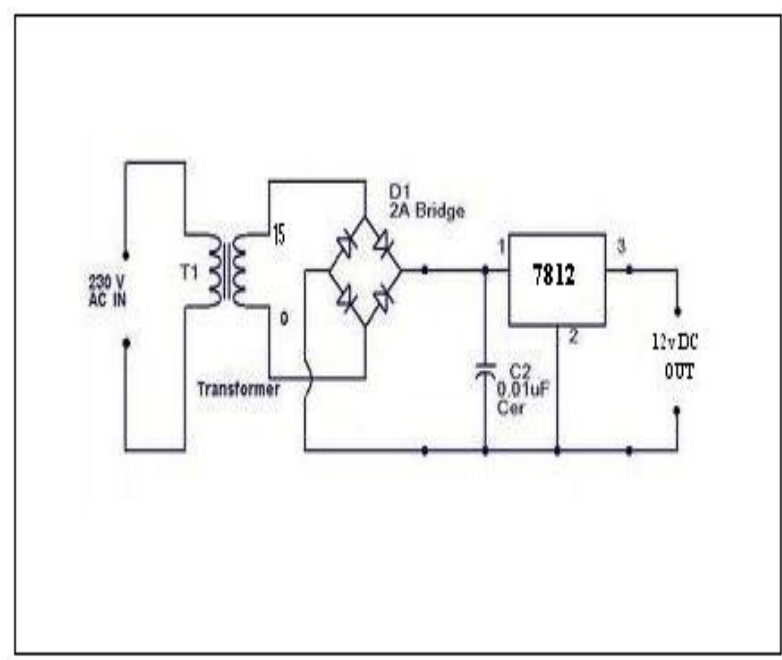

Fig 2.2: Regulated power supply using 7812

3.3.2. $12 v$ with $1.2 A$ current: In this supply we are used regulator LM317 which have following specifications:

- Adjustable DC output voltage

- Guaranteed 1.5A current

- Output is short circuit protected

- Current limit constant with temperature

This is used to drive the Stepper motor through driver circuit.

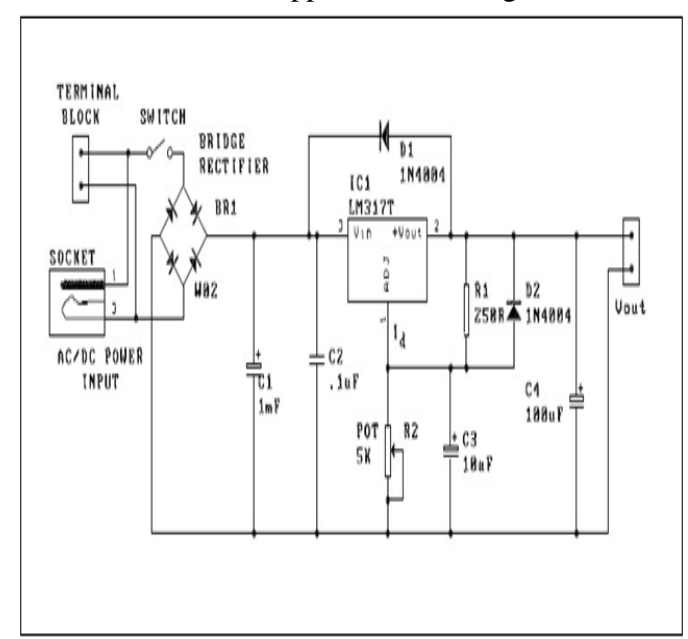

Fig 2.3: Adjustable power supply using LM317 


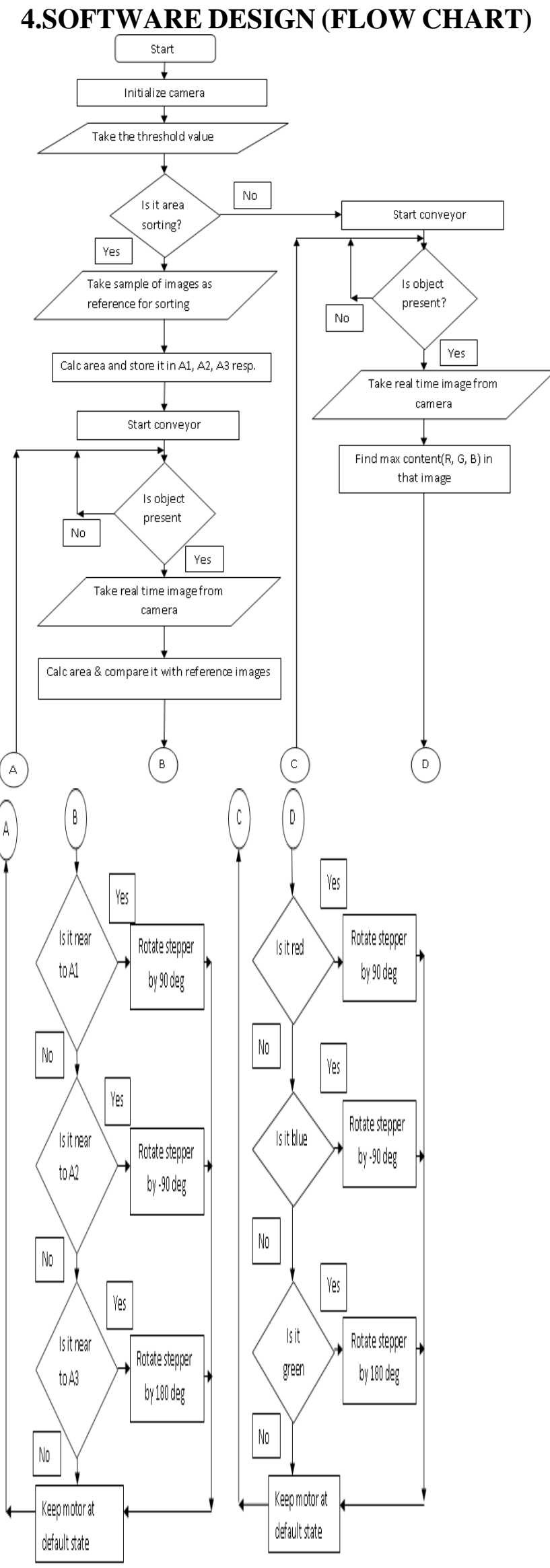

Fig 2.3: Flow Chart

\section{ENTIRE SYSTEM OPERATION}

Object sorting is done using two ways: Color wise sorting and Size wise sorting.

\subsection{Color-Wise Sorting}

5.1.1. Image Acquisition: First the camera is initialized. Then the conveyor is started, and the system is in poling mode to check whether the object is infornt of camera or not. If the object is in front of camera, then the image is captured through webcam using getsnapshot function.

5.1.2. Color Sorting: Here the captured image of the object is processed to find out which color of object is? This decision is made by separating the R, G, B content of captured image. Then the sum command is used to find the maximum content present in the captured image. [6]

5.1.3. Execution of placing assembly: In this step, according to the count the stepper motor steps. If the RED content is high then stepper motor steps +90 degree through parallel port \& driver circuit. If the BLUE content is high, then stepper motor steps -90 degree through parallel port \& driver circuit. If the GREEN content is high, then stepper motor steps +180 degree through parallel port \& driver circuit. After completion of each step (+90 deg, $-90 \mathrm{deg},+180 \mathrm{deg}$ ) motor comes to the default Position

\subsection{Size-Wise Sorting}

5.2.1. Image Acquisition: Firstly, the camera is initialized \& the appropriate thresholding values are set. Then the reference images given for comparison. For all the reference images, the area is calculated and stored in A1, A2, A3 variables. After this the conveyor is started to check the object is in front of camera or not. If the object is in front of camera then the image is captured through webcam and thresholding is done.

5.2.2. Object sorting using area: Here, first the area of the captured image is calculated. This area is then compared with the stored area A1, A2, A3. According to that the command is given to the placing assembly

5.2.3. Execution of placing assembly: In this step, according to the count the stepper motor steps. If the area is matched with A1 then count is 14 and the stepper motor steps +90 degree. If the area is matched with A2 then count is -14 and the stepper motor steps -90 degree If the area is matched with A3 then count is 26 and the stepper motor steps +180 degree t. After completion of each step motor rests to the default Position.

\section{RESULT AND DISCUSSION}

The output module consists of the two assemblies:

- Conveyor Assembly

- $\quad$ Stepper motor Assembly

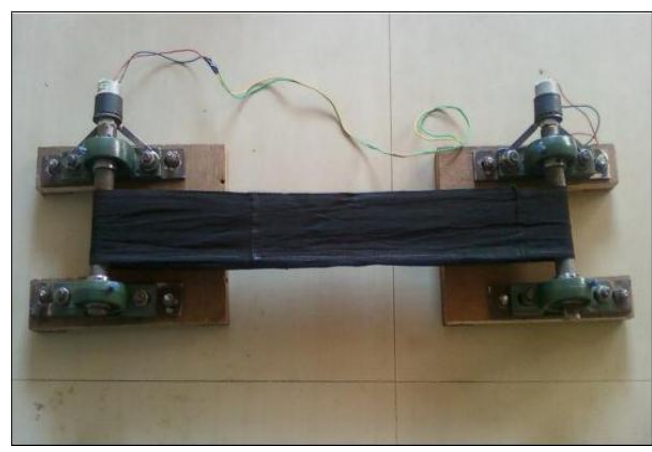

Fig 3.1: Conveyor Assembly 


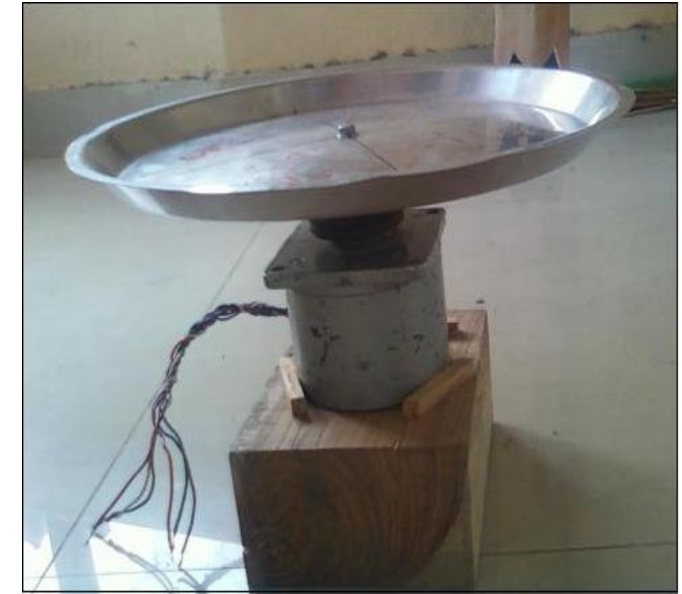

Fig 3.2: Stepper Motor Assembly

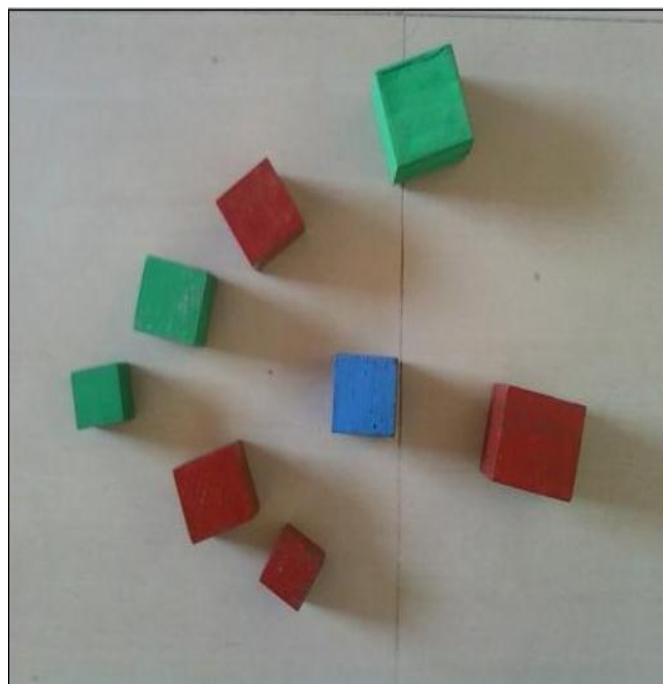

Fig 3.3: Objects used for sorting

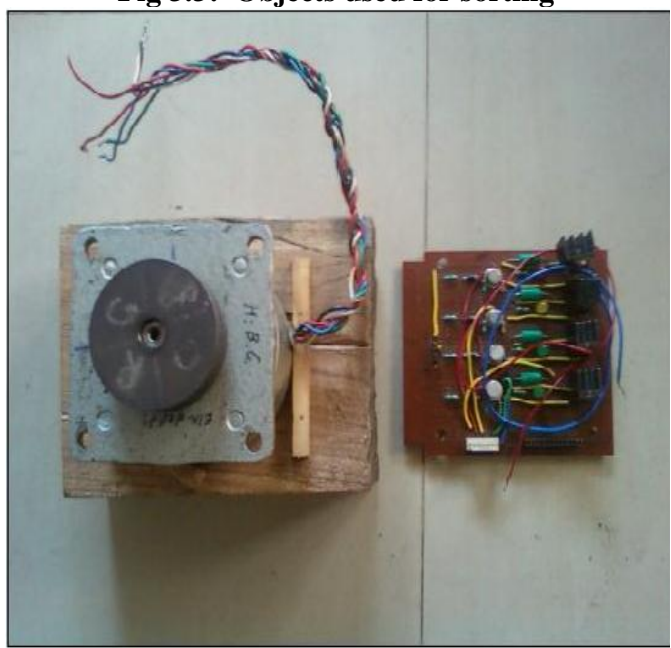

Fig 3.4: Stepper Motor with driver Circuit.

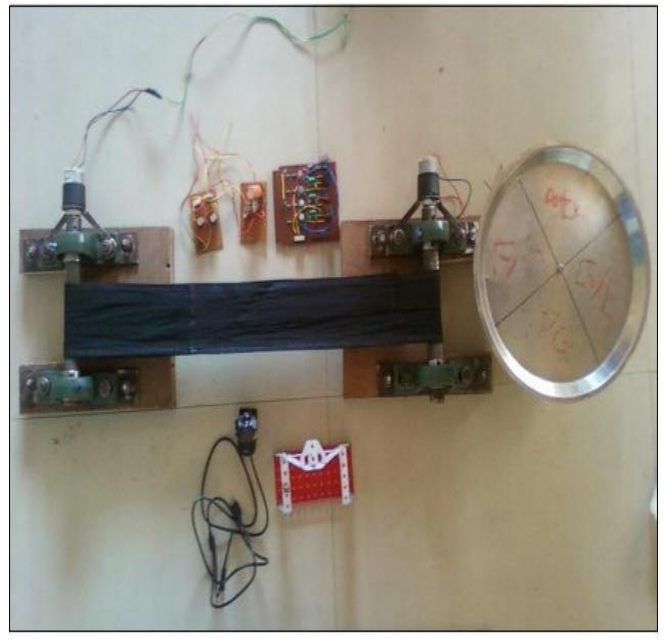

Fig 3.5: Entire System

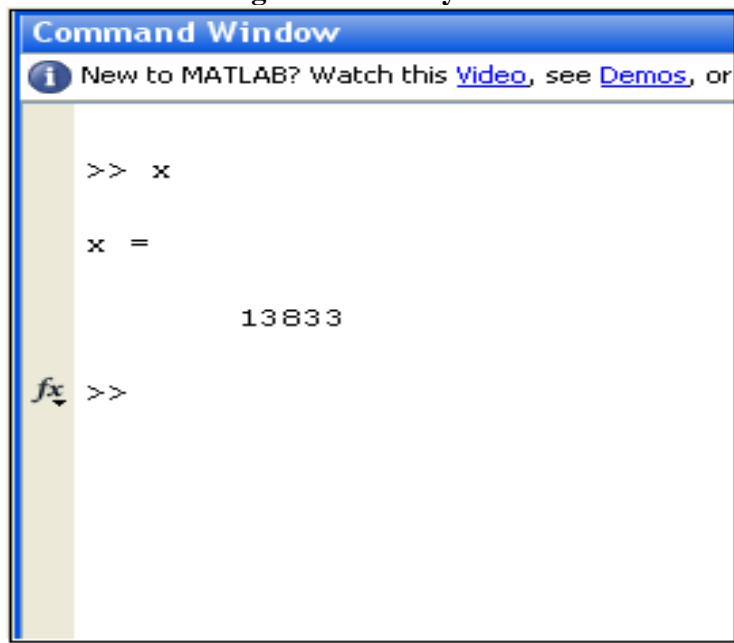

Fig 3.6: Area of Object

Command Window $\quad+1 x x$

(1) New to MATLAB? Watch this Yideo, see Demos, or read Gettinn Started.

Warning: This Parallel adaptor ('parallel') will not be provided in future releases

of Data Icquisition Toolbox. Instead, it will be available as a separate domload.

See Solution 1-5Ligod for details.

matched with second object

stepper motor steps -90 degree

stepper motor is at default position $\mid x \rightarrow>>$

Fig 3.7: Sorting of Object according to Area 


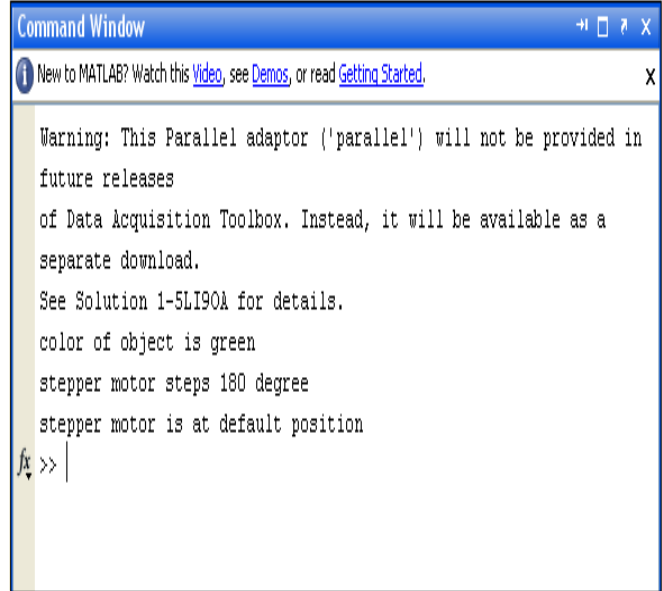

Fig 3.8: Sorting of Object according to Color

\section{APPLICATIONS}

A. In small scale and large scale industries. To sort the products based on the various parameters.

B. Can be used in store department.

C. In malls and small shops(e.g. hardware)

D. In medicals and wine industries to sort the bottles of various sizes

E. In food industries to identify the rotten fruits.

\section{CONCLUSION}

Hence, an object sorting system for domestic/industrial control has developed using the concepts of Image Processing, Robotics Mechanism and parallel communication without help of DSP processor. The model developed is user friendly.

We conclude that for fast manipulation the algorithm implemented in the MATLAB ${ }^{\circledR}$ is suitable for our sorting problem. Result of sorting the object may not work for 100 percent but it is working for nearly 90 to $94 \%$ in case of our algorithm. It can be improved by increasing the accuracy and it depends on atmospheric factors.
Faster manipulation and efficient sorting can be done by using DSP processor and LAB-VIEW ${ }^{\circledR}$. So our goal project has been met to recognize gesture effectively.

\section{REFERENCES}

[1] Arko Djajadi, Fiona Laoda, Rusman Rusyadi, Tutuko Prajogo, Maralo Sinaga," A model vision of sorting system application using robotic manipulator" TELKOMNIKA: Indonesian Journal of Electrical Engineering, ISSN: 16936930, e-ISSN: 2087-278X _ 137accredited by Directorate General of Higher Education of Indonesia, SK No: 51/Dikti/Kep/201

[2] http://www.vision-systems.com

[3] S. Md. Iqbal, D. Ganesan and Dr. P. Sudhakara Rao, "Mechanical System for On-line Fruits Sorting and grading using Machine Vision Technology", J. Instrum. Soc. India 34 (3) $153-162$

[4] www.mathworks.com

[5] http://students.iitk.ac.in/roboclub/data/tutorials

[6] Rafael C. Gonzalez and Richard E. Woods, "Digital Image Processing", Third Edition, Vol.3, Pearson Publication.

[7] Mohammad Osiur Rahman ,M A Hannan, Edgar Scavino,Aini Hussain ,Hassan Basri ," An efficient paper grade identification method for automatic recyclable waste paper sorting" European Journal of Scientific Research, Vol.25 No.1 (2009), pp.96-103.

[8] M. Khojastehnazhand, M. Omid* and A. Tabatabaeefar, "Development of a lemon sorting system based on color and size", African Journal of Plant Science Vol. 4(4), pp. 122127, April 2010 ,ISSN 1996-0824 (C) 2010 Academic Journals 